\title{
The Accurate Method for Removing Misalignment Errors from Testing Rectangular Optics
}

\author{
Zhu Li \\ Purification Equipment \\ Research Institute of Handan \\ Handan,056027, China \\ E-mail:lzhu718@126.com
}

\author{
Xiaokun Wang \\ Key Laboratory of Optical System \\ Advanced Manufacturing Technology \\ Changchun Institute of Optics, Fine \\ Mechanics and Physics, Chinese Academy \\ of Sciences \\ Changchun,130033, China \\ E-mail:jimwxk@sohu.com
}

\author{
Song Li \\ Key Laboratory of Optical System \\ Advanced Manufacturing Technology \\ Changchun Institute of Optics, Fine \\ Mechanics and Physics, Chinese \\ Academy of Sciences \\ Changchun,130033, China \\ E-mail:xz_zhuli@126.com
}

\begin{abstract}
The limitations of current interferogram reduction software with Zernike polynomials in analyzing rectangular interferogram are illuminated. The Zernike polynomials are no longer orthogonal over an rectangular region and have no explicit physical meanings. The reasonable mathematical model for removing translation errors is established based on least squares fitting. The experiment is carried on with a $200 \mathrm{~mm} \times 75 \mathrm{~mm}$ aspherical mirror by null-compensation, the misalignment errors can be eliminated from the original phase distribution exactly, thus the accurate surface map is gained, so it provides a guarantee for the superprecision fabrication.
\end{abstract}

Keywords - rectangular optics; Zernike polynomial; misalignment errors; least squares fitting

\section{INTRODUCTION}

RC system and Gregory system both have advantages of simple structure, colorless and so on[1]. Therefore, these two mirror systems have been used widely in field of the largediameter telescope, infrared or ultraviolet system. However, there is generally a central obscuration on the primary mirror of the co-axial two-mirror system, while the system is astigmatic. Off-axis three mirror anti-elimination astigmatic system(TMA) has advantages of fewer components, unobstructed, wide band, strong ability of inhibiting stray light, high modulation transfer function, so the system has become a new development trend of large field of view of the long focal length optical system[2-5]. The primary mirror and third mirror in the TMA system are generally rectangular aspherical elements, so the rectangular interferogram will be attained in the detection.

Usually Zernike polynomials are used in the commercial interferometer data processing software to describe the aberrations ${ }^{[6]}$. Zernike polynomials have several interesting properties.They are one of an infinite number of complete sets of polynomials in two real variables, that are orthogonal in a continuous fashion over the interior of a unit circle. It is important to note that the Zernikes are orthogonal only in a continuous fashion over the interior of a unit circle, and in general they will not be orthogonal over a discrete set of data points within a unit circle.

Because Zernike polynomials are weighted orthogonal in the unit circle, while the coefficients of the polynomials can be connected with the coefficient of Seidel aberrations. When testing circular optical elements, phase distribution can be expressed well with Zernike polynomials and there are clear physical meanings to the corresponding terms. Usually the first four (Piston, X-tilt and Y-tilt and the Power) are removed as an adjustment error in the test results. However there will be a certain degree of error if Zernike polynomials are still used to describe the surface shape in the detection of the rectangular optical elements. Because the Zernike polynomials are not orthogonal in a rectangular domain and there is no longer a clear physical meaning while there will be coupling phenomenon between its coefficients. Based on least squares fitting, a reasonable method of removing adjusting error is put forward in the article and the exact processing residuals distribution are obtained, thus a guarantee is provided to the ultra-precision machining.

\section{BASIC THEORY}

Using Interference method to measure a rectangular optical component, if the high order errors of the system can be ignored, the phase measurement values of the wavefront error can be expressed as:

$$
w(x, y)=e(x, y)+\varepsilon(x, y)
$$

Where $e(x, y)$ is the error of the test optical surface, $\varepsilon(x, y)$ is the positioning error. Usually four positioning errors are introduced in the alignment process, corresponding to the tilts for the $\mathrm{x}$ direction and the $\mathrm{y}$ direction, defocus and piston, that is,

$$
\varepsilon(x, y)=a x+b y+c\left(x^{2}+y^{2}\right)+d
$$

Using the least squares fitting, the optimal solution of the alignment coefficients can be calculated:

$$
\left[\begin{array}{l}
a \\
b \\
c \\
d
\end{array}\right]=\left[\begin{array}{cccc}
\sum x x & \sum x y & \sum x\left(x^{2}+y^{2}\right) & \sum x \\
\sum y x & \sum y y & \sum y\left(x^{2}+y^{2}\right) & \sum y \\
\sum\left(x^{2}+y^{2}\right) x & \sum\left(x^{2}+y^{2}\right) y & \sum\left(x^{2}+y^{2}\right)^{2} & \sum\left(x^{2}+y^{2}\right) \\
\sum x & \sum y & \sum\left(x^{2}+y^{2}\right) & n
\end{array}\right]^{-1}
$$




$$
\left[\begin{array}{c}
\sum x w \\
\sum y w \\
\sum\left(x^{2}+y^{2}\right) w \\
w
\end{array}\right]
$$

Where $\mathrm{n}$ is the number of sampling points. Error coefficients a, b, c and d can be calculated from the above equation. Accurate residual distribution of the test optical components can be attained by reducing the error from $w$ thus completing the measurements to the entire surface shape.

\section{EXPERIMENT}

First Null compensation test is applied to a aperture of $200 \mathrm{~mm} \times 75 \mathrm{~mm}$ aspheric mirror with Zygo digital interferometer. The detection principle is shown in Figure1.The plane wave or spherical wave from interferometer is converted to an aspherical wave in coincident with the shape of the asphere surface through the compensator, so all the lights incident vertically to the asphere tested and return along the same route, forming interference fringes with the reference wavefront[7-8].

The null corrector can introduce enough aberration (of the opposite sign) into the test beam so that it eliminates the aberration produced by testing the aspheric surface at its center of curvature[9-10]. It consists of two positive pianoconvex lenses: the field and relay lenses. Both lenses are located near the center of curvature of the mirror under test, so they are significantly smaller than the test mirror itself. The function of the field lens is to rearrange in a linear way the rays that arrive to the relay lens. The function of the relay lens is to introduce the bulk of the optical correction and to obtain a null test point.

The distribution of the surface shape and the interferogram are shown in Figure 2 and Figure 3. Analyzed by the interferometer data processing software MetroPro, the distribution of phase after reducing the alignment errors of piston, tilt, and defocus is shown in Fig 4.The PV value: $0.359 \lambda$, the RMS value: $0.041 \lambda$.Using Matlab computing software to finish the program of removing error, leastsquares fitting to deal with the interference data, reducing the adjustment errors, accurate surface error distribution is obtained as shown in Fig 5.The PV value of the surface phase distribution: $0.355 \lambda$ RMS value: $0.038 \lambda$. The adjustment error coefficients obtained by analysis and calculation are shown in Table I .

TABLE I. COMPARISON OF THE MISALIGNMENT COEFFICIENTS

\begin{tabular}{|l|l|l|}
\hline & $\begin{array}{l}\text { Coefficients calculated } \\
\text { by MetroPro }\end{array}$ & $\begin{array}{l}\text { Coefficients calculated by } \\
\text { least squares fitting }\end{array}$ \\
\hline Piston & 1.783 & 1.764 \\
\hline X Tilt & -0.461 & -0.452 \\
\hline Y Tilt & -1.416 & -1.435 \\
\hline Power & 0.038 & 0.071 \\
\hline
\end{tabular}

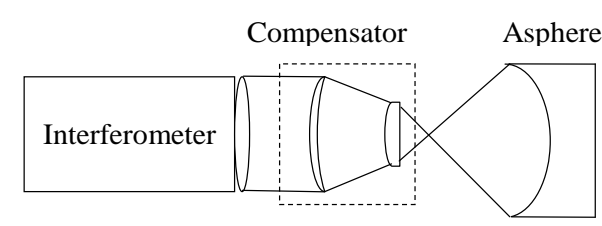

Figure 1. Schematic of testing asphere by null-compensation
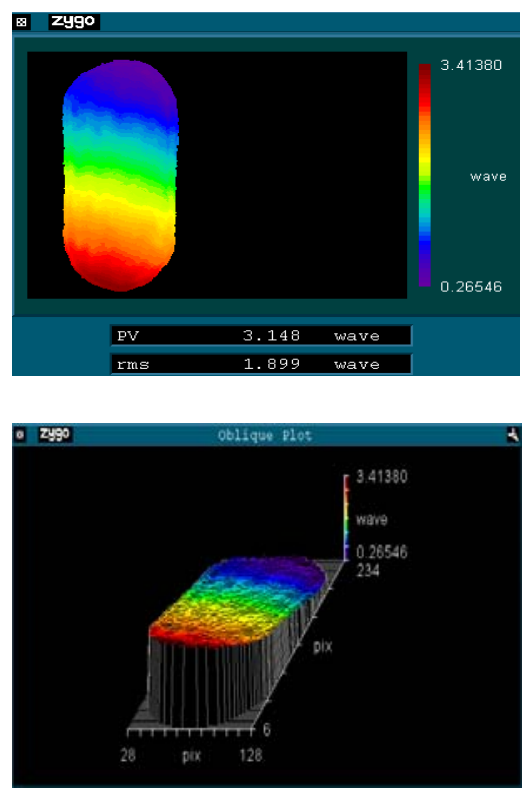

Figure 2. Experiment results for a rectangular mirror before removing misalignment errors

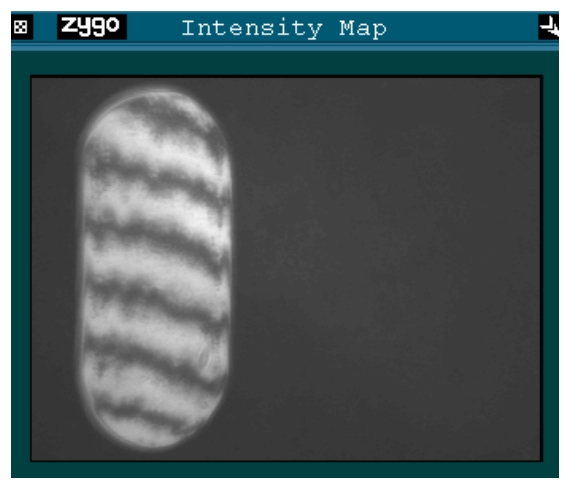

Figure 3. Interferogram of the asphere 


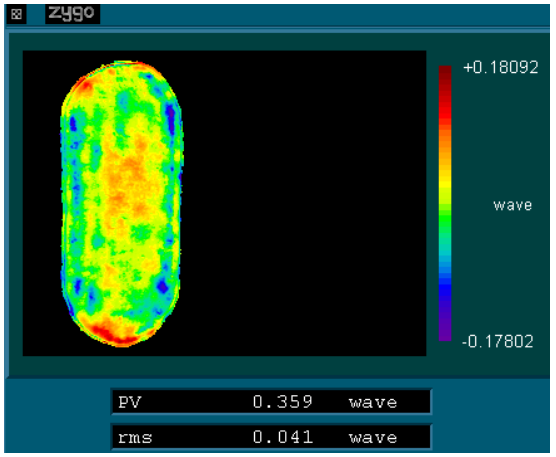

Figure 4. Surface map after removing piston,tilt and power by misalignment MetroPro software

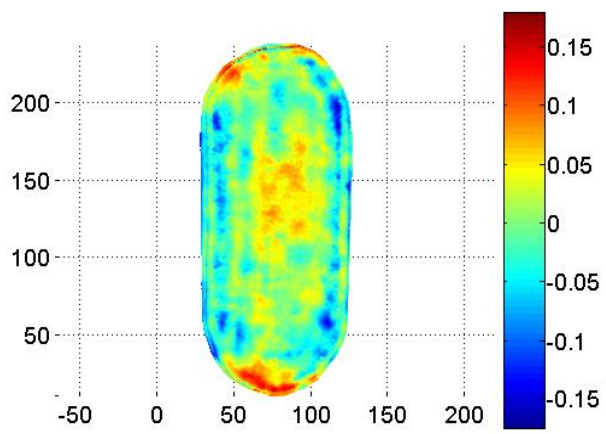

Figure 5. Surface map after removing errors by least squares fitting

\section{ANALYSIS AND CONCLUSIONS}

The Zygo interferomer is mounted on a X/Y/Z translation stage. The null lens is mounted on a adjustor with 5 dofs, which can adjust the tip, tilt and $\mathrm{X} / \mathrm{Y} / \mathrm{Z}$ translation. The tested asphere is mounted on a three axes stage, which can adjust the tip, tilt and defocus of the asphere precisely. The whole set up is mounted on a vibration isolator, and the testing temperature is $22^{\circ} \mathrm{C}$.

According to the comparison and analysis, there are some differences between the error coefficients obtained by interferometer MetroPro software and the least squares fitting. There are also some differences between the phase distributions and PV, RMS values after separating the adjustment errors. This is because usually rectangle interferometer is dealt with as a circular domain in the interferometer data processing software to finish Zernike polynomial fitting and also treated as a circular domain for the calculation of Seidel aberration coefficients. The coefficients obtained in this way must be different with the coefficients calculated by Zernike polynomial fitting. It's not reasonable to remove the first four terms as adjustment errors. Using least squares method to fit the interference pattern of the rectangle, adjustment errors can be excluded with higher precision and a more accurate distribution of surface can be obtained. This method is also applicable to the removal of other non-circular optical element adjustment error, providing means to the ultra-finishing and testing.

\section{REFERENCES}

[1] J.H. Pan, Design, fabrication and testing of optical aspheres, Beijing: Science Press, 1994.

[2] X.J. Zhang, "Manufacturing of a Three Mirror Anastigmat Telescope," SPIE, vol. 4829, pp. 884-885, 2003.

[3] T. Zobrist, J. Burge, and H. Martin. "Laser tracker surface measurements of the 8.4 m GMT primary mirror segment," SPIE, vol. 7426, pp. 742613-1, 2009.

[4] M.Q. Wang, Z.H. Wang, and J.G. Bai , "Optical design of off-axis three-mirror anastigmatic system for imaging spectrometer ," Infrared and Laser Engineering, vol. 41, pp. 167-172, 2012.

[5] D.D. Walker, A.T.H. Beaucamp, and R.G. Bingham, "The Precessions Process for Efficient Production of Aspheric Optics for Large Telescopes and Their Instrumentation," Proceedings of SPIE, vol. 4842, pp. 73-84, 2003.

[6] D. Malacara, Optical Shop Testing, 2nd ed. New York: J.Wiley\&Sons, 1992.

[7] F. Zhang, "Testing of rectangle aperture off-axis aspheric mirror in process of computer controlled manufacturing," Optical Technique, vol. 28, pp. 289-290, 2002.

[8] P.J. Guo, and J.C. Yu, "Design and certification of a null corrector to test hyperboloid convex mirror," SPIE, vol. 6150, pp. 259-263, 2006.

[9] J.M. Sasian, S.A. Lerner, and J.H. Burge, "Design, tolerancing, and certification of a null corrector to test 8.4 meter mirrors," Proc SPIE, vol. 3739, pp. 444-449, 1999.

[10] Beraud, J. Espiard, and R. Geyl, "Optical figuring and testing of the VLT 8.2-m primary mirrors," SPIE, vol. 2536, pp. 413-420, 1995. 